\title{
Regulation of Hydroxycinnamic Acid Degradation Drives Agrobacterium fabrum Lifestyles
}

\author{
Thibault Meyer, ${ }^{1}$ Sébastien Renoud, ${ }^{1}$ Armelle Vigouroux, ${ }^{2}$ Aurélie Miomandre, ${ }^{1}$ Vincent Gaillard, ${ }^{1}$ \\ Isabelle Kerzaon, ${ }^{1}$ Claire Prigent-Combaret, ${ }^{1}$ Gilles Comte, ${ }^{1}$ Solange Moréra, ${ }^{2}$ Ludovic Vial, ${ }^{1}$ and \\ Céline Lavire ${ }^{1,+}$ \\ ${ }^{1}$ Université de Lyon, F-69622, Lyon, France; Université Lyon 1, Villeurbanne, France; CNRS, UMR5557, Ecologie Microbienne, \\ Villeurbanne, France; INRA, UMR1418, Villeurbanne, France; and ${ }^{2}$ Institute for Integrative Biology of the Cell (I2BC), CNRS \\ CEA Univ. Paris-Sud, Université Paris-Saclay, Avenue de la Terrasse, Gif-sur-Yvette 91198, France
}

Accepted 16 February 2018.

\begin{abstract}
Regulatory factors are key components for the transition between different lifestyles to ensure rapid and appropriate gene expression upon perceiving environmental cues. Agrobacterium fabrum C58 (formerly called $A$. tumefaciens C58) has two contrasting lifestyles: it can interact with plants as either a rhizosphere inhabitant (rhizospheric lifestyle) or a pathogen that creates its own ecological niche in a plant tumor via its tumorinducing plasmid (pathogenic lifestyle). Hydroxycinnamic acids are known to play an important role in the pathogenic lifestyle of Agrobacterium spp. but can be degraded in A. fabrum species. We investigated the molecular and ecological mechanisms involved in the regulation of $A$. fabrum species-specific genes responsible for hydroxycinnamic acid degradation. We characterized the effectors (feruloyl-CoA and $p$-coumaroyl-CoA) and the DNA targets of the MarR transcriptional repressor, which we named HcaR, which regulates hydroxycinnamic acid degradation. Using an hcaR-deleted strain, we further revealed that hydroxycinnamic acid degradation interfere with virulence gene expression. The HcaR deletion mutant shows a contrasting competitive colonization ability, being less abundant than the wild-type strain in tumors but more abundant in the rhizosphere. This supports the view that $A$. fabrum C58 HcaR regulation through ferulic and $p$-coumaric acid perception is important for the transition between lifestyles.
\end{abstract}

Bacteria can adopt different lifestyles and adapt to multiple habitats because of specific pathways whose expression is tightly controlled (Brooks et al. 2011; Cases et al. 2003; White

${ }^{\dagger}$ Corresponding author: C. Lavire; E-mail: celine.lavire@univ-lyon1.fr

Funding: T. Meyer, A. Vigouroux, L. Vial, S. Moréra, and C. Lavire were supported by the CNRS (Mission pour l'interdisciplinarité, Agromics 20142016). Part of this work was initiated by the EcoGenome project of the French Agence Nationale de la Recherche (grant ANR-BLAN-08-0090) and by the French national program EC2CO-Biohefect/Ecodyn//Dril/MicrobiEn (IBAD). T. Meyer received a doctoral grant from the French Ministère de l'Education Nationale, de l'Enseignement Supérieur et de la Recherche. This work benefited from the I2BC microcalorimetry platform, supported by FRISBI ANR-10-INSB-05-01, and from the DTAMB, the "Centre Technologique des Microstructures" and "Serre and chambres climatiques" platforms, supported by the FR BioEnviS Research Federation.

*The $\boldsymbol{e}$-Xtra logo stands for "electronic extra" and indicates that five supplementary figures and four supplementary tables are published online.

(c) 2018 The American Phytopathological Society et al. 2011). Indeed, a given pathway can be beneficial in one lifestyle but deleterious in another (Andersson and Hughes 2010; Price et al. 2013). The transition between lifestyles involves a coordinated modification of gene expression upon signal perception to express the appropriate gene at the right time (Duprey et al. 2014; Valentini et al. 2018). However, the presence of a regulatory system selected for its effectiveness in one environment can have different consequences (either beneficial or deleterious) in environments submitted to different constraints (Schick et al. 2015). Therefore, one may wonder whether regulatory systems are mainly optimized for bacteria to adapt to different lifestyles or are specialized for one of them.

Agrobacterium fabrum (one of the species of the A. tumefaciens complex) strain C58 is a suitable model to study the relative fitness cost of a regulator in contrasting environments. This bacterium has two lifestyles: (i) as a nonpathogen rhizosphere colonizer or (ii) as a pathogen that causes crown-gall disease by creating its own ecological niche in a plant tumor via its tumorinducing plasmid (pTi) (Nester 2015). Hydroxycinnamic acids (HCA) play an important role in the pathogenic lifestyle of Agrobacterium spp. (Guo et al. 2017; Joubert et al. 2002). Briefly, a wounded plant releases amino acids, sugars, and phenolic compounds such as acetosyringone and HCA that contribute to guiding Agrobacterium spp. to the wounded site by chemotaxis (Ashby et al. 1988; Guo et al. 2017). Moreover, HCA trigger the expression of pTi-encoded virulence (vir) genes responsible for T-DNA transfer to the plant host cell nuclei and its insertion into the plant genome which, in turn, results in crown gall formation (Bhattacharya et al. 2010; Bolton et al. 1986; Kalogeraki et al. 1999). Among vir genes, virH2 encodes an O-demethyl transferase, thought to detoxicate HCA-like ferulic acid and to be involved in the negative control of vir gene induction (Brencic et al. 2004). However, the circular chromosome of A. fabrum contains a species-specific gene cluster named SpG81-b (i.e., a region present in bacteria of this species but absent from strains of other Agrobacterium spp.) also involved in HCA degradation (Lassalle et al. 2011). The SpG8-1b region is composed of eight genes from atul415 to atul422 (hcaR); it enables bacteria to utilize HCA as a carbon source (Fig. 1) (Campillo et al. 2014). In addition, HCA degradation induces the transcription of the SpG8$1 \mathrm{~b}$ region, probably through HCA degradation intermediates (Baude et al. 2016).

The uncharacterized regulatory protein HcaR (Atu1422), annotated as a multiple antibiotic resistance regulator (MarR), could be involved in the repression of SpG8-1b gene cluster expression in the absence of HCA (Lassalle et al. 2011). This protein belongs to the MarR family, among which regulatory 
proteins involved in the repression of HCA degradation genes have been described recently in other soil microorganisms (Kim et al. 2016; Otani et al. 2016). These repressors can interact with different ligands: HCA (ferulic and $p$-coumaric acids) or CoAactivated HCA ( $p$-coumaroyl-CoA and feruloyl-CoA). At the onset of the study of HcaR of A. fabrum C58, we had no clue whether SpG8-1b genes were expressed in interaction with plants (in the rhizosphere or in tumors). However, we suspected that regulation of HCA degradation from the SpG8-1b region would be more important for bacterial competitiveness in the tumor than in the rhizosphere environment; thus, we investigated the molecular and ecological roles of HcaR using biochemical and in planta approaches. The present article describes how HcaR regulates SpG8-1b gene expression and how HCA degradation intermediates can release HcaR from DNA binding. We also demonstrate that, in contrast to rhizosphere colonization, repression of HCA degradation by HcaR is important for providing A. fabrum a competitive advantage in colonizing tumors and is required to induce vir genes upon ferulic acid perception. HcaR regulation could be decisive for the transition between the rhizospheric and pathogenic lifestyles.

\section{RESULTS}

\section{HCA degradation: Comparison}

between the $\mathbf{C 5 8}$ wild-type and $\mathrm{C58 \Delta hcaR}$ strains.

Degradation of different HCA in the C58 and C584hcaR strains was compared by measuring the remaining HCA in the growth culture supernatant after $8 \mathrm{~h}$ by high-performance liquid chromatography (HPLC) (Fig. 2). Acetosyringone and acetovanillone were not degraded by either strain. Ferulic acid, p-coumaric acid, caffeic acid, and 3,4-dimethoxycinnamic acid were more degraded in the mutant than in the wild type $(10,6.5$, 1 , and $2 \%$ left, as compared with 59, 22, 63, and 100\% left in the wild type, respectively). Interestingly, some HCA such as cinnamic acid, 4-methoxycinnamic acid, $o$-coumaric acid, and $m$-coumaric acid were only degraded by the C584hcaR strain
(11, 21, 32, and $21 \%$ left, as compared with $97,98,97$, and $98 \%$ left in the wild type, respectively).

\section{Ferulic acid and $p$-coumaric acid modulate the expression of SpG8-1b genes.}

Plasmid reporter gene fusions were constructed for the transcription units of the SpG8-1b region (Fig. 1) and introduced into the wild-type strain in order to evaluate gene expression in response to HCA. Compared with the fluorescence level measured in the absence of ferulic acid (Table 1), the fluorescence level measured in the presence of ferulic acid was significantly higher (i.e., 2.36-, 1.68-, 1.29-, and 1.10-fold for atu1416, atu1417, atu1418, and hcaR reporter fusions, respectively). Leaving aside atu1418, which is not involved in $p$-coumaric acid degradation (Fig. 1), similar results were obtained with $p$-coumaric acid (Table 2; Supplementary Table S4). Acetovanillone, caffeic acid, 3,4-dimetoxycinnamic acid, cinnamic acid, $m$-coumaric acid, $o$-coumaric acid, and 4-methoxycinnamic acid had no inductive effect on atu1416, atu1417, atu1418, or hcaR reporter fusions (Table 2). Overall, these results indicate that only ferulic and $p$-coumaric acids induced the expression of SpG8-1b HCAdegradation genes.

\section{The MarR transcriptional regulator HcaR is a repressor of SpG8-1b gene expression.}

The plasmid reporter fusions of SpG8-1b gene expression were introduced into the C58 4 hcaR strain; then, the fluorescence level was measured in the absence or in the presence of ferulic acid. In the C58 4 caR mutant strain, the atu1416, atu1417, and hcaR genes were constitutively expressed (a high fluorescence level was measured even in the absence of ferulic acid). Moreover the fluorescence level measured for the atu1416, atu1417, and hcaR reporter fusions were two to six times higher in the C58 4 hcaR mutant strain than in the wildtype strain in either the absence or presence of ferulic acid (Table 1). In contrast, the fold changes between C58 $\Delta$ hcaR and the wild-type strains were similar for the atul418 gene fusion and the empty pOT1e vector (Table 1). These results show that
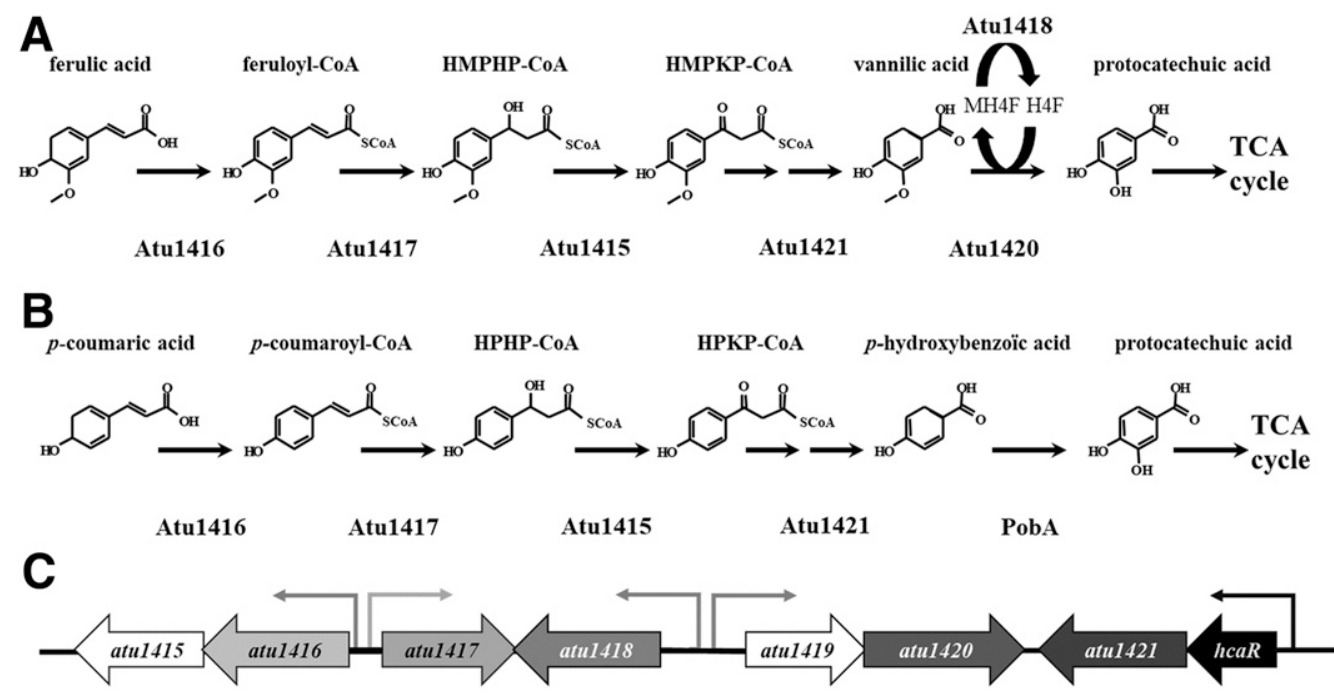

Fig. 1. Ferulic acid and p-coumaric acid degradation pathway and organization of the SpG8-1b region. A, Ferulic acid degradation pathway. A coenzyme A is added to ferulic acid by Atu1416, a feruloyl-CoA synthase. Feruloyl-CoA is then converted by enoyl-CoA hydratase Atu1417 into 4-hydroxy-3-methoxyphenyl$\beta$-hydroxypropionyl (HMPHP)-CoA which, in turn, is transformed into vanillic acid by Atu1415, a phenylhydroxypropionyl-CoA dehydrogenase, and then Atu1421, a 4-hydroxy-3-methoxyphenyl- $\beta$-ketopropionyl-CoA (HMPKP)-CoA $\beta$-keto-thiolase. Atu1418 and Atu1420 degrade vanillic acid into protocatechuic acid. The $p c a$ genes, described by Parke $(1995,1997)$, allow the integration of this latter molecule into the TCA cycle. B, $p$-Coumaric acid degradation pathway. The same enzymes catalyze the transformation of $p$-coumaric acid into $p$-coumaroyl-CoA, then 4-hydroxy-phenyl- $\beta$-hydroxypropionyl (HPHP)-CoA, 4-hydroxy-phenyl$\beta$-ketopropionyl-CoA (HPKP)-CoA, and p-hydroxybenzoic acid. The latter is converted into protocatechuic acid by PobA (Atu4544) (Parke 1995, 1997). C, Organization of the SpG8-1b region. The atu1415-atu1416, atu1419-atu1420, and hcaR-atu1421 genes belong to a different transcription unit. Promoters are shown with small arrows indicating the direction of gene transcription. 
HcaR is a transcriptional repressor that regulates atu1416, atu1417, and its own transcription.

\section{DNA-binding target and affinity.}

In order to characterize the DNA-binding target of HcaR, we purified HcaR, which appeared as a dimer in solution according to gel filtration measurements. Using the intergenic regions (approximately $300 \mathrm{bp)} \mathrm{of} \mathrm{atu1416-1417,} \mathrm{atu1418-1419,} \mathrm{hcaR,}$ and atu2645 (gene outside the SpG8-1b region) as DNA probes for binding assays (Fig. 3A), we showed that HcaR bound to the atu1416-1417 and hcaR DNA probes but not to the atu2645 and atu1418-1419 DNA probes. These results demonstrate that HcaR acts as a transcriptional repressor by targeting the atu1416-1417 and $h c a R$ intergenic regions.

To further characterize bound DNA, in silico comparison of the promoter regions of atu1416-1417 and hcaR was performed. We identified a 25-bp conserved region containing 16 identical base pairs (Fig. 3B). The region contained two inverted repeated sequences overlapping the -35 box regulatory element of atu1416-1417 whereas, in the hcaR promoter sequence, the inverted repeated sequence imperfectly overlapped the -10 and -35 regulatory elements (Fig. 3B). In order to verify that HcaR binds to the conserved DNA region, we also performed gel-shift assays using two 170-bp probes located either downstream $\left(\mathrm{P}_{\text {Down }}\right)$ or upstream $\left(\mathrm{P}_{\mathrm{Up}}\right)$ of the conserved region (Supplementary Fig. S1). Purified HcaR bound to the probe containing the 25-bp motif whereas $\mathrm{P}_{\text {Down }}$ and $\mathrm{P}_{\mathrm{Up}}$ did not.

The mean $\mathrm{K}_{\mathrm{D}}$ value between HcaR and the conserved DNA region used for gel-shift experiments determined by isothermal titration microcalorimetry (ITC) was $49 \pm 9 \mu \mathrm{M}$. ITC data revealed a 2:1 binding stoichiometry (one HcaR dimer binds one DNA molecule, as given from $n=0.53 \pm 0.15$ ), and demonstrated a positive enthalpy change of $16.180 \pm 555.4 \mathrm{cal} / \mathrm{mol}$ upon DNA binding (Fig. 3D).

\section{HcaR effectors are feruloyl-CoA and $p$-coumaroyl-CoA.}

To identify whether ferulic acid or ferulic acid intermediate degradation compounds were the effectors of HcaR, the plasmid reporter fusions of SpG8-1b gene expression

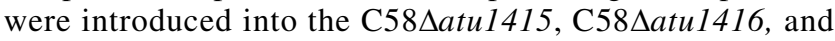
C58 4 atu 1417 mutant strains, and fluorescence levels were measured in the presence or absence of ferulic acid (Supplementary Table S3). In C584atu1416, which did not degrade ferulic acid (Fig. 1), supplementation with ferulic acid had no effect on gene induction (basal fluorescence), indicating that this compound was not the effector. In the C58 4 atu 1415 strain, the degradation process stopped at the 4-hydroxy-3methoxyphenyl- $\beta$-hydroxypropionyl-CoA stage (Fig. 1), and the fluorescence level of the atu1416 and atu1417 reporter fusions was greater in the presence of ferulic acid, suggesting that feruloylCoA or 4-hydroxy-3-methoxyphenyl- $\beta$-hydroxypropionyl-CoA could be the effectors. In the C58 $\Delta$ atu1417 strain, in which feruloyl-CoA accumulates (Fig. 1), expression of the atu1416, atu 1417, and hcaR reporter gene fusions increased by 5.1-, 3.7-, and 1.4-fold, respectively, as compared with expression in the absence of ferulic acid. In addition, this induction level was greater than in C58. Overall, these results show that feruloylCoA was responsible for the induction of atu1416, atu1417, and $h c a R$ gene expression.

Using gel-shift assays, we verified the ability of feruloyl-CoA to relieve the interaction between HcaR and the conserved intergenic region. As expected, ferulic acid had no effect on protein-DNA binding, unlike feruloyl-CoA (Fig. 3C). Similar results were obtained with $p$-coumaroyl-CoA (Supplementary Fig. S2). Therefore, feruloyl-CoA and $p$-coumaroyl-CoA are the effectors of HcaR and they activate protein release from DNA.

Table 1. Ferulic acid degradation effect on the SpG8-1b gene expression after $24 \mathrm{~h}$ of incubation

\begin{tabular}{|c|c|c|c|}
\hline Genes & C58 & C58 $\Delta h c a R$ & Fold change $^{a}$ \\
\hline \multicolumn{4}{|l|}{ Patu1416 } \\
\hline $0 \mu \mathrm{M}$ & $6.12 \pm 0.37$ & $40.53 \pm 1.73$ & 6.62 \\
\hline $750 \mu \mathrm{M}$ & $14.43 \pm 0.48$ & $45.06 \pm 2.89$ & 3.12 \\
\hline \multicolumn{4}{|l|}{ Patu1417 } \\
\hline $0 \mu \mathrm{M}$ & $4.23 \pm 0.32$ & $18.39 \pm 0.61$ & 4.35 \\
\hline $750 \mu \mathrm{M}$ & $7.13 \pm 0.45$ & $18.64 \pm 0.82$ & 2.61 \\
\hline \multicolumn{4}{|l|}{ Patu1418 } \\
\hline $0 \mu \mathrm{M}$ & $13.01 \pm 0.49$ & $10.19 \pm 0.35$ & 0.78 \\
\hline $750 \mu \mathrm{M}$ & $16.8 \pm 1.32$ & $13.5 \pm 0.56$ & 0.80 \\
\hline \multicolumn{4}{|l|}{ PhcaR } \\
\hline $0 \mu \mathrm{M}$ & $6.41 \pm 0.43$ & $14.73 \pm 0.63$ & 2.30 \\
\hline $750 \mu \mathrm{M}$ & $7.06 \pm 0.40$ & $14.00 \pm 0.73$ & 1.98 \\
\hline \multicolumn{4}{|c|}{ Empty pOT1e } \\
\hline $0 \mu \mathrm{M}$ & $5.25 \pm 0.32$ & $4.06 \pm 0.15$ & 0.77 \\
\hline $750 \mu \mathrm{M}$ & $5.41 \pm 0.34$ & $4.07 \pm 0.19$ & 0.75 \\
\hline
\end{tabular}

${ }^{a}$ Numbers in bold indicate significant differences of fluorescence values (Mann-Whitney-Wilcoxon test, $P=0.05$ ) between both strains.

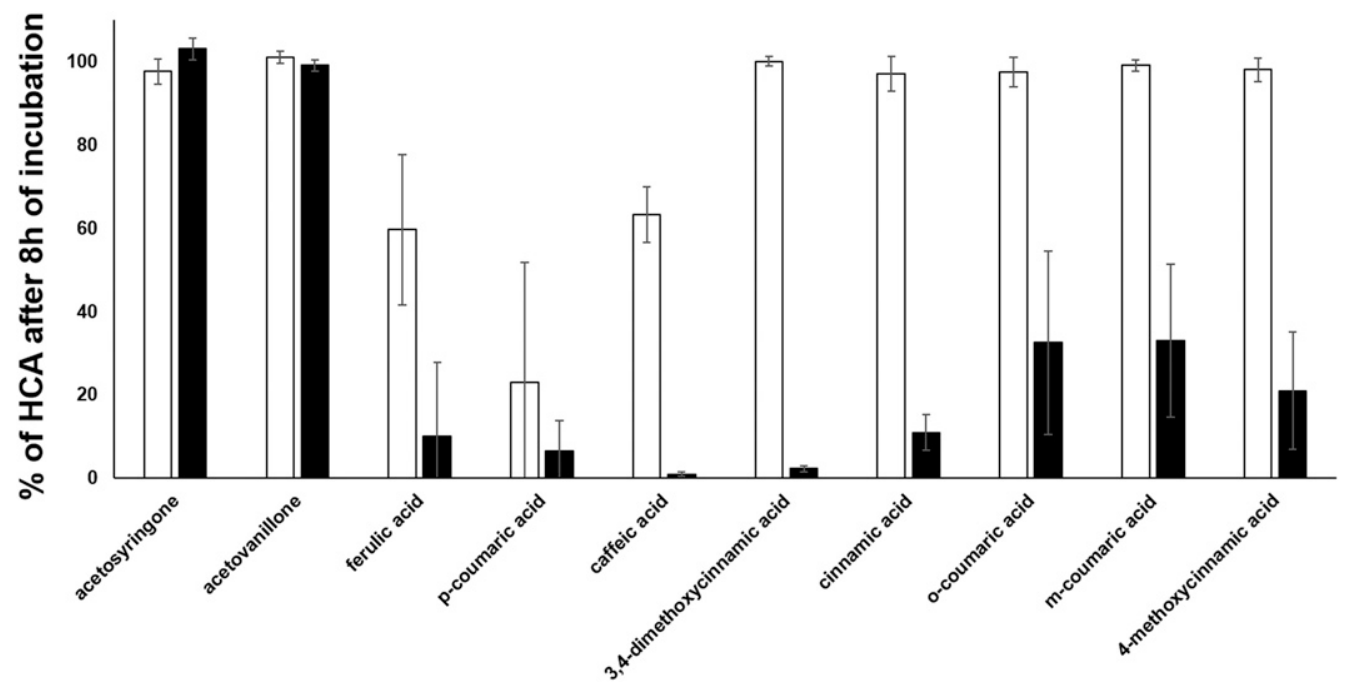

Fig. 2. Abundance of hydroxycinnamic acid (HCA) compounds in the wild-type and C58 4 caR mutant strains. The abundance of different HCA potentially degraded by the SpG8-1b pathway was measured in culture supernatant in both strains. The presence of the supplemented compounds was quantified by highperformance liquid chromatography after $8 \mathrm{~h}$ of incubation and compared with their concentration prior to incubation $\left(\mathrm{T}_{0}=100 \%\right)$. Compound degradation is represented in white for the wild-type strain and in black for the C584hcaR strain. 


\section{hcaR deletion modifies $\operatorname{vir} B$ gene expression.}

HCA are considered to be inducers of vir genes; therefore, we compared the ability of strains C58, C58 $\Delta$ SpG8-1b (deleted for $h c a R$ and all genes involved in HCA degradation-no HCA degradation), and C584hcaR (hcaR-deleted-constitutive HCA degradation) to induce $\operatorname{vir} B$ gene expression, using a plasmid reporter fusion (virB-egfp transcriptional fusion) (Fig. 4). Acetosyringone, which is not degraded by C58 (Fig. 2), induced fluorescence in the strains, which shows that HcaR is not directly involved in vir gene expression. In strain C58, a significant fluorescence level was detected in the presence of ferulic acid, confirming that this compound induced $\operatorname{vir} B$ gene expression (Fig. 4). In the C58 $\Delta$ SpG8-1b mutant, the fluorescence level in the presence of ferulic acid was higher than in the wild type. In the C584hcaR mutant, however, no fluorescence signal was measured (Fig. 4), probably due to the rapid degradation of ferulic acid (Fig. 2).

\section{Implications of HcaR regulation in $A$. fabrum lifestyles.}

The C58 and C58 4 hcaR strains were individually inoculated into plant stems. Both strains induced tumors and colonized the stems similarly (Supplementary Fig. S3), even though expression of vir genes differed between the two strains. Indeed, fewer bacteria were detected based on enhanced green fluorescent protein (eGFP) fluorescence in C58 4 haR (Supplementary Fig. $\mathrm{S} 4)$. In a tomato tumor generated after inoculation with the C584hcaR mutant strain containing an atu1416 plasmid reporter fusion, all the bacteria within the tumor expressed HCA degradation genes (constitutive expression: yellow cells, Fig. 5A; Supplementary Fig. S5). In contrast, in strain C58, among

Table 2. Ratio of reporter gene induction in the presence or absence of different hydroxycinnamic acids after 24 h of incubation ${ }^{\text {a }}$

\begin{tabular}{|c|c|c|c|c|c|c|c|c|}
\hline Gene & Acetovanillone & $\begin{array}{l}p \text {-Coumaric } \\
\text { acid }\end{array}$ & $\begin{array}{l}\text { Caffeic } \\
\text { acid }\end{array}$ & $\begin{array}{l}\text { 3.4-Dimethoxycinamic } \\
\text { acid }\end{array}$ & $\begin{array}{l}\text { Cinnamic } \\
\text { acid }\end{array}$ & $\begin{array}{l}m \text {-Coumaric } \\
\text { acid }\end{array}$ & $\begin{array}{l}o \text {-Coumaric } \\
\text { acid }\end{array}$ & $\begin{array}{l}\text { 4-Methoxycinnamic } \\
\text { acid }\end{array}$ \\
\hline Patu1416 & 0.99 & 5.70 & 0.85 & 0.92 & 0.93 & 1.13 & 1.07 & 1.08 \\
\hline Patu1417 & 1.12 & 3.50 & 0.80 & 0.89 & 0.94 & 1.13 & 0.99 & 1.16 \\
\hline Patu1418 & 1.02 & 0.90 & 0.91 & 0.84 & 0.93 & 1.02 & 0.86 & 1.02 \\
\hline PhcaR & 1.18 & 1.68 & 0.89 & 0.89 & 1.02 & 1.06 & 1.02 & 1.15 \\
\hline $\begin{array}{l}\text { Empty } \\
\text { pOT1e }\end{array}$ & 1.13 & 1.04 & 0.91 & 0.80 & 0.95 & 1.12 & 1.00 & 1.09 \\
\hline
\end{tabular}

${ }^{a}$ Numbers in bold indicate significant differences of fluorescence values (Mann-Whitney-Wilcoxon test, $P=0.05$ ) between both strains.

A HcaR
(pmol)
C

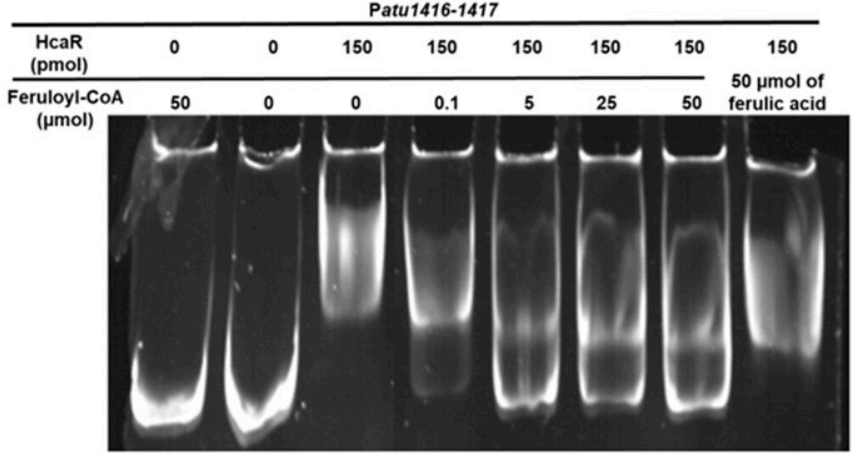

B

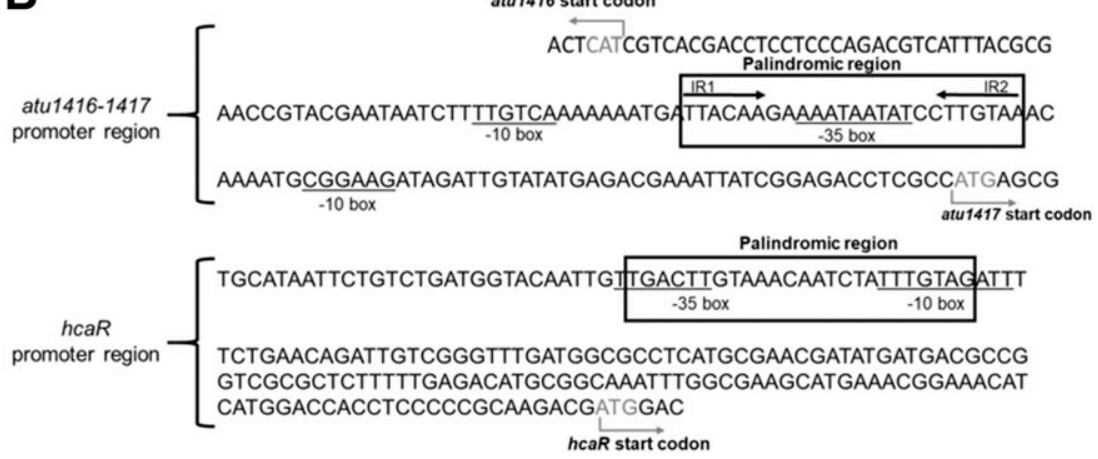

D

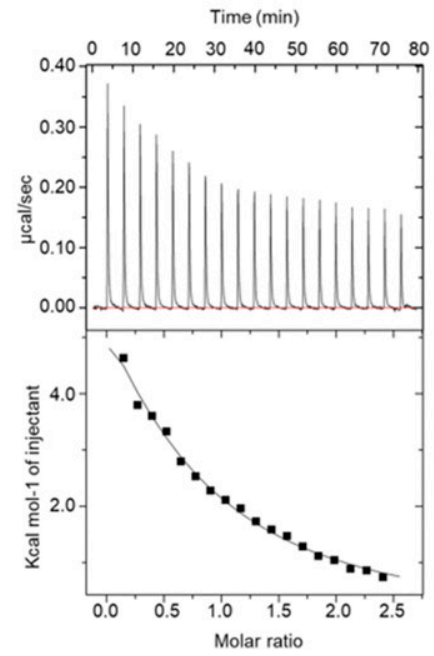

Fig. 3. HcaR regulation mechanism: DNA binding and identification of HcaR as an effector. A, Gel-shift analysis of HcaR binding to the promoter regions of SpG8-1b. B, In silico analysis of the atu1416-1417 and hcaR promoter regions: -10 and -35 boxes are highlighted, palindromic regions are framed, and inverted repeat sequences are indicated by arrows. C, Gel-shift analysis of HcaR binding to the Patu1416-1417 region, with feruloyl-CoA at different concentrations $(0.1$ to $50 \mu \mathrm{M})$ or with $50 \mu \mathrm{M}$ ferulic acid (last well). D, The top graph of the HcaR microcalorimetry experiment shows heat differences following injection of the DNA ligand (top panel) and integrated heats of injection with the best fit (solid line) to a single binding model using MicroCal Origin (lower panel). 
the cells present inside the tumor (red or yellow cells), only a few expressed the HCA degradation genes (yellow cells, Fig. $5 A$ ). The ability of the two strains to efficiently colonize the tumor was tested in a 1:1 competitive experiment. hcaR deletion had no deleterious effects in vitro (measured competitive index $[\mathrm{CI}]=1.18$, not significantly different from $1, P=0.96$ ) but, in the tumor tissue, C584hcaR was less abundant than C58 $\left(\mathrm{CI}=0.66\right.$, significantly lower from $\left.1, P=4.7 \times 10^{-6}\right)$ (Fig. 5C).

The C58 and C584hcaR strains were individually inoculated on tomato seed. Both strains grew similarly in the rhizosphere. In the plants inoculated with either the C58 or the C584hcaR strain containing an atu1416 fusion reporter plasmid, most cells expressed HCA degradation genes (yellow-green, Fig. 5C). However, 15 days after seed coinoculation (1-1), C584hcaR was more abundant in the tomato rhizosphere than $\mathrm{C} 58(\mathrm{CI}=$ 4.18 , significantly different from $1, P=8,7 \times 10^{-6}$ ). These data reveal differences in the expression of HCA degradation genes and opposite effects of HcaR regulation in the two plant environments; namely, the tumor and the rhizosphere.

\section{DISCUSSION}

In this study, we addressed the molecular mechanism of the species-specific A. fabrum HcaR regulator and its ecological role in the two contrasting lifestyles of Agrobacterium. Indeed, HCA is degraded through the expression of the species-specific chromosomal gene cluster and is also involved in the initiation of the plasmid-encoded pathogenicity program.

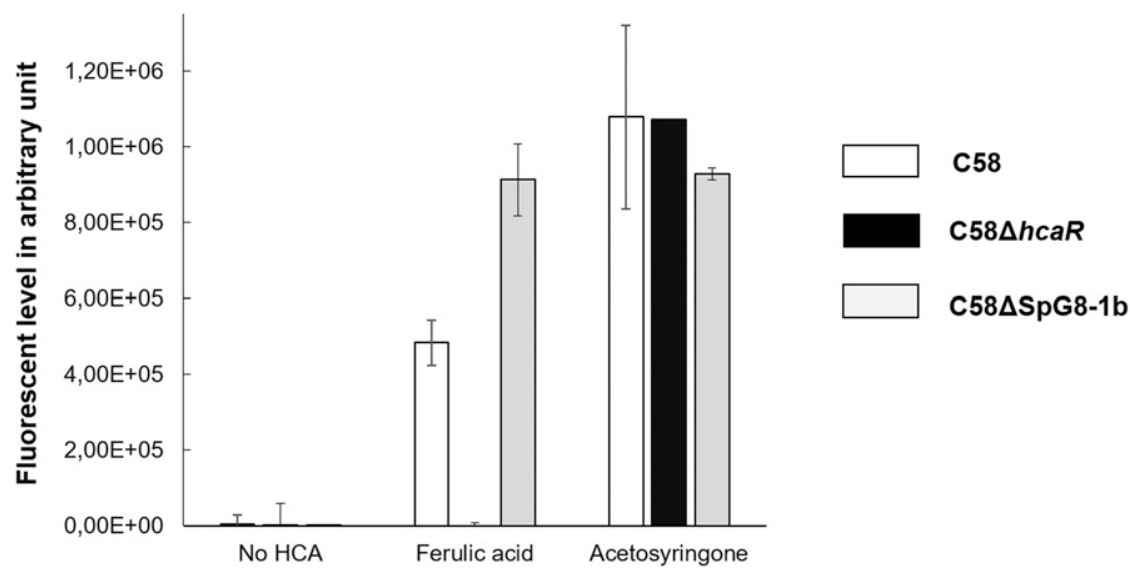

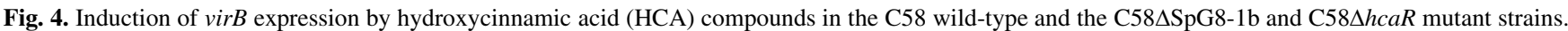
Induction of $v i r B$ in C58 (white squares), $\mathrm{C} 58 \Delta \mathrm{SpG} 8-1 \mathrm{~b}$ (gray squares), or C58 $\Delta h c a R$ (black squares) cultured in culture medium supplemented with $750 \mu \mathrm{M}$ ferulic acid or acetosyringone was monitored by measuring fluorescence. Fluorescence levels (in arbitrary units) were corrected by dividing fluorescence values by values of the optical density at $600 \mathrm{~nm}$, and then subtracting the fluorescence value of the empty pOT1e vector. Acetosyringone, which is not degraded by the SpG8-1b pathway, was used as a positive control of virB induction.
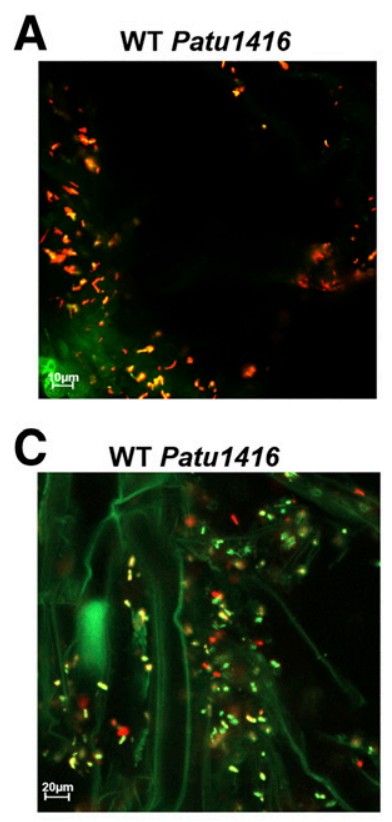
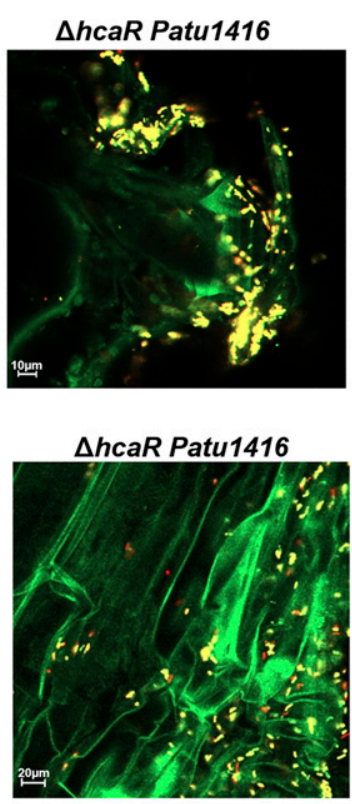

B

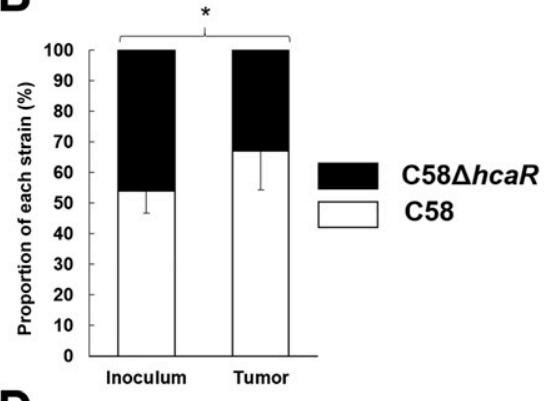

D

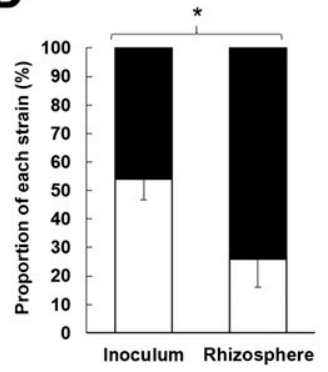

Fig. 5. Effect of $h c a R$ deletion on plant colonization and on the expression of hydroxycinnamic acid (HCA) degradation genes in planta. Expression of Patu1416 reporter $\mathbf{A}$ and $\mathbf{C}$, fusions and $\mathbf{B}$ and $\mathbf{D}$, fitness were compared between C58 (wild type [WT], white) and C584hcaR (mutant, black) in a tomato tumor (upper part) and in the rhizosphere (lower part). Representative pictures from three biological replicates and two independent experiments are shown (A and C). Red fluorescence from M-cherry indicates the presence of bacteria, while green fluorescence corresponds to the area of Patu1416 induction. Yellow fluorescence shows bacteria that were both active and able to express Patu1416. Plant autofluorescence is represented in green and the scale in white. The proportion of each simultaneously inoculated strain was calculated in the tumor and the rhizosphere after 3 and 2 weeks of growth, respectively (B and D). 
We characterized HcaR as a transcriptional repressor of the MarR family (Deochand and Grove 2017; Grove 2017; Perera and Grove 2010), which is dimeric in solution and binds to a 25-nucleotide palindromic sequence overlapping the -10 or -35 box. DNA-HcaR binding represses its own transcription and that of the first genes involved in the HCA degradation pathway. This type of regulation is consistent with regulation mechanisms triggered by regulators of the MarR family, whereby the repressor prevents gene transcription by binding to DNA sequences in promoter regions until an effector releases it from DNA to allow gene transcription (Grove 2017). For the MarR regulator of Acinetobacter sp. ADP1, which regulates HCA degradation genes, the following ligands can abolish its DNA repression: ferulic acid, $p$-coumaric acid, 3,4-dihydroxybenzoate, and vanillin (Kim et al. 2016; Parke and Ornston 2003). In contrast, we identified only two effectors of HcaR in A. fabrum C58, which are different because they are CoA derivatives of ferulic acid and of $p$-coumaric acid.

Hence, the effectors (feruloyl-CoA or p-coumaroyl-CoA) that enhance the expression of the degradation pathway are the products of the first step of this pathway (feruloyl-CoA synthase, Atu1416). This involves a low basal expression of atu1416 to create a positive feedback loop in the presence of HCA. The same ligands have been associated with CouR and FerR regulators from Rhodococcus sp. and Rhodopseudomonas palustris, respectively (Hirakawa et al. 2012; Otani et al. 2014; Pan et al. 2015). Nonetheless, the DNA-binding motif recognized by $A$. fabrum C58 HcaR differs from any other MarR regulator (Calisti et al. 2008; Hirakawa et al. 2012; Kim et al. 2016; Otani et al. 2016). Although we showed that HcaR is activated by two HCA derivatives, this protein can regulate the degradation of numerous HCA (Fig. 2). Indeed, HCA that are not able to induce SpG8-1b gene expression (Table 2) can be degraded by proteins of the HCA degradation pathway (C584hcaR; Fig. 2). That is why we named it HcaR. Interestingly, the HCA degradation pathway is indirectly induced by ferulic acid and $p$-coumaric acid, which are the major constituents of plant cell walls. Moreover, ferulic acid released by wounded plant cells is one of the chemical signals that induces the Agrobacterium pathogenic program (Brencic et al. 2004; Joubert et al. 2002; McCullen and Binns 2006). The presence of this compound leads to the expression of vir genes (Nester 2015). Additionally, a pTi-encoded HCA degradation protein (VirH2) transforms ferulic acid into caffeic acid. This has been suggested to reduce the metabolic cost of overexpressing the pathogenic machinery by controlling vir gene expression, because caffeic acids do not induce vir gene expression (Brencic et al. 2004). Thus, HCA degradation gene regulation through $\mathrm{HcaR}$ could interfere with pathogenesisrelated gene expression.

In the present study, the virB gene was poorly expressed in the C58 4 hcaR mutant both in vitro in the presence of ferulic acid (Fig. 4) and in planta. In contrast, vir genes are expressed in strain C58 and in the C58 $\Delta$ SpG8-1b mutant, which contains neither HcaR nor the degradation genes (Fig. 4). These observations imply that HcaR is not directly involved in vir gene activation. Hence, a rapid degradation of the vir gene inducer (ferulic acid) due to constitutive expression of HCA degradation genes in the C58 4 hcaR mutant does influence the expression of vir genes. Thus, it is tempting to speculate that HcaR might play an important role in the pathogenic lifestyle. According to the usual method to test Agrobacterium pathogenicity (i.e., inoculation of pure culture on injured tomato stems) (Vaudequin-Dransart et al. 1995), HcaR is not crucial for pathogenicity. Indeed, both the wild type and the C584hcaR mutant were able to form tumors. Nonetheless, several key steps of Agrobacterium pathogenicity (for example chemotaxis and attachment) (Heindl et al. 2014; Merritt et al. 2007) are bypassed by our inoculation method and one cannot exclude that HcaR would play a role in a more natural context.

Regulation by a MarR regulator has been proposed to confer a fitness advantage to bacteria in the absence of HCA in order to avoid synthesis of unnecessary enzymes (Grove 2017). HCA are not uniformly distributed in plant tissues, nor are they consistently available for bacteria (Mandal et al. 2009; Simoh et al. 2009). Because this system induces SpG8-1b gene expression after HCA detection, it could be advantageous for bacteria to avoid the metabolic cost of constitutively expressing HCA degradation genes in plant environments with a low HCA content. In a pathogenic context, our findings are consistent with this hypothesis. In tumor tissue, HCA are linked to malate and formed a stock of hydroxycinnamoyl-malate (Simoh et al. 2009). Hence, strain C58 can adapt its metabolism via HcaR regulation according to HCA availability, as shown by in planta expression analyses (Figs. 5A and C). A fitness study of hcaR-deleted C58 4 caR demonstrated that this regulation mechanism was advantageous because C58 outcompeted the mutant strain. The disadvantage of the C58 4 hcaR mutant strain could be linked to a metabolic cost associated with the constitutive expression of HCA degradation genes in a low-HCA environment.

Analyses of the importance of HcaR regulation in a rhizospheric context gave opposite results, because the C58 4 hcaR mutant outcompeted strain C58 in the rhizosphere (Fig. 5D). Similarly, a competitive advantage conferred by HCA degradation during colonization of the tomato rhizosphere has been reported in Ralstonia solanacearum (Lowe et al. 2015). In our case, because the rhizosphere is rich in free HCA (Mandal et al. 2009), it seemed important for bacteria to achieve competitive rhizosphere colonization not only to degrade HCA, as both strains did, but also to rapidly assimilate a broad range of HCA, as the C58 4 caR mutant did.

In summary, we showed that HcaR regulation influences expression of vir genes that are important and influences the execution of the program necessary for pathogenesis. Moreover, it seems relevant to repress HCA degradation genes in the pathogenic context and to rapidly express them in the rhizospheric context. Feruloyl-CoA and $p$-coumaroyl-CoA are the HcaR effectors that allow a positive feedback loop and induce HCA degradation gene expression. Thus, more than being involved in the pathogenicity itself, HcaR regulation through ferulic acid perception may be important for the transition between $A$. fabrum rhizospheric and pathogenic lifestyles.

\section{MATERIALS AND METHODS}

\section{Bacterial strains and growth conditions.}

The bacteria and plasmids used for this study are listed in Supplementary Table S1. Escherichia coli were grown routinely, with shaking $(150 \mathrm{rpm})$, at $37^{\circ} \mathrm{C}$ in Luria-Bertani (LB) medium. Growth media were supplemented with appropriate antibiotics (tetracycline, $10 \mu \mathrm{g} / \mathrm{ml}$; gentamicin, $15 \mu \mathrm{g} / \mathrm{ml}$; and ampicillin, $100 \mu \mathrm{g} / \mathrm{ml}$ ) when necessary. The $A$. fabrum strains were grown with shaking $(160 \mathrm{rpm})$, at $28^{\circ} \mathrm{C}$ in yeast peptone glucose (YPG)-rich medium or in AT minimal medium supplemented with $10 \mathrm{mM}$ succinate and $10 \mathrm{mM}$ ammonium sulfate (Campillo et al. 2012). AT minimal medium was supplemented with $750 \mu \mathrm{M}$ HCA or phenolic compounds as required, and with the appropriate antibiotic (gentamicin, $20 \mu \mathrm{g} / \mathrm{ml}$ ). Phenolic compounds (i.e., acetosyringone, acetovanillone, ferulic acid, $p$-coumaric acid, caffeic acid, 4-dimethoxycinnamic acid, cinnamic acid, 4-methoxycinnamic acid, $o$-coumaric acid, and $m$-coumaric acid) were obtained from Sigma-Aldrich (St. Louis). HCA-CoA derivatives were obtained from TransMIT PlantMetaChem (Giessen, Germany). 
1A medium was used to select $A$. fabrum strains after in planta inoculation (Shams et al. 2012).

\section{Construction of the deletion mutant and transcriptional fusions.}

The C584hcaR strain was constructed by recombination with a suicide vector (pJQ200sk vector, Quandt and Hynes 1993) according to a strategy previously described by Lassalle et al. (2011). The vector containing the recombinant region (amplified by polymerase chain reaction [PCR]), flanking downstream and upstream of the hcaR gene, was introduced into A. fabrum C58 by electroporation. Single-crossover integration was selected by gentamycin resistance on YPG medium plates. Gentamycinresistant colonies were spread on YPG plates containing 10\% sucrose to obtain plasmid excision and double-crossover events. hcaR deletion was confirmed by PCR analysis and DNA sequencing (GenoScreen, Lille, France).

eGFP transcriptional fusions were generated as follows. The promoter regions of the $\operatorname{virB}$ and SpG8-1b genes (namely, PvirB, Patu1415, Patu1416, Patu1417, Patu1418, and PhcaR) were amplified with specific primers listed in Supplementary Table S2 and cloned into pGEM-T Easy vectors prior to subcloning of the ClaI-SalI fragment into the reporter plasmid pOT1e (Allaway et al. 2001) digested with the same enzymes. pOT1eM was obtained by cloning $m$-cherry under Tac promoter control (constitutive expression) into the pOT1e vector digested with ClaI and SalI (Habbadi et al. 2017). The previously described promoter regions were ligated to the pOT1eM vector in the SpeI restriction site. Constructions were confirmed by PCR using pOT1eF and pOT1eR primers and by sequencing. Reporter constructions were introduced into A. fabrum C58 wild-type and mutant strains by electroporation, and gentamycin-resistant colonies were selected.

\section{Measurement of promoter activity.}

Expression of the different genes involved in HCA degradation was measured in A. fabrum with a pOT1e plasmid harboring an eGFP transcriptional fusion (Allaway et al. 2001). Fluorescence was quantified in microplates filled with $200 \mu \mathrm{l}$ of AT medium and supplemented with phenolic compounds at a final concentration of $750 \mu \mathrm{M}$. Microplate wells were inoculated with overnight cultures to obtain an optical density at $600 \mathrm{~nm}\left(\mathrm{OD}_{600 \mathrm{~nm}}\right)$ equal to 0.2 . Microplate readings were performed with a Xenius spectrofluorometer (SAFAS-Monaco, Monaco, Monaco), after 14 or $24 \mathrm{~h}$ of incubation at $28^{\circ} \mathrm{C}$. The following parameters were used: absorbance at $600 \mathrm{~nm}$, fluorescence excitation at $488 \mathrm{~nm}$, and emission at $510 \mathrm{~nm}$. Results were normalized by dividing them by the $\mathrm{OD}_{600 \mathrm{~nm}}$ values and then they were compared with values from the empty pOT1e vector. Fluorescence levels were compared using the Wilcoxon rank sum test adjusted with Bonferroni method $(P=0.05)$, and computed with the "vegan" package in the R v3.1.3 statistical software environment (R Core Team 2014).

\section{Cloning, expression, and purification of HcaR.}

The hcaR gene was PCR amplified with the primer pair (petHcaRFXhoI-petHcaRFXbaI). The product was digested with $X b a \mathrm{I}-X h o \mathrm{I}$ and ligated into the pET29a plasmid digested with the same enzymes. E. coli C41 strain was transformed by the recombinant plasmid. For HcaR purification, cells were grown at $37^{\circ} \mathrm{C}$ in $\mathrm{LB}$ medium with kanamycin until they reached an $\mathrm{OD}_{600 \mathrm{~nm}}$ value of 0.6 , then supplemented with $0.5 \mathrm{mM}$ isopropyl- $\beta$-D-thiogalactoside to induce HcaR production. After centrifugation, cells were suspended in $50 \mathrm{mM}$ Tris- $\mathrm{HCl}(\mathrm{pH} 8.0)$ and $300 \mathrm{mM} \mathrm{NaCl}$, and disrupted by sonication. After centrifugation at $25,000 \times g$ for $30 \mathrm{~min}$ at $4{ }^{\circ} \mathrm{C}$, each supernatant was loaded onto a HiTrap talon column (GE
Healthcare, Uppsala, Sweden). Protein elution was performed with $50 \mathrm{mM}$ Tris- $\mathrm{HCl}$ ( $\mathrm{pH} 8.0), 300 \mathrm{mM}$ imidazole, and $150 \mathrm{mM} \mathrm{NaCl}$. The fractions containing HcaR were loaded onto a gel filtration column (HiLoad 26/60 Superdex 200 prep grade; GE Healthcare) equilibrated with $50 \mathrm{mM}$ Tris- $\mathrm{HCl}(\mathrm{pH}$ 8.0) and $150 \mathrm{mM} \mathrm{NaCl}$. The protein fractions were pooled, concentrated, and stored at $-80^{\circ} \mathrm{C}$.

Gel-shift assay. HcaR binding was determined by gel-shift assays. The intergenic regions of atu1416-1417, atu1418-1419, and $h c a R$ were amplified with specific primer pairs. The atu2645 intergenic region was used as a nonspecific control probe. Gel-shift assays were performed at $28^{\circ} \mathrm{C}$ in a $10-\mu \mathrm{l}$ reaction mixture containing $0.3 \mathrm{pmol}$ of the DNA probe, $2 \mu \mathrm{l}$ of $5 \times$ binding buffer (Invitrogen, Carlsbad, CA), 150 pmol of HcaR protein, and 0 to $50 \mu \mathrm{M}$ aromatic compounds. HcaR-free controls were included. After incubation for $30 \mathrm{~min}$ at room temperature, $2 \mu \mathrm{l}$ of EMSA gel-loading solution (Invitrogen) was added, and the samples were separated by electrophoresis on $6 \%$ polyacrylamide (29:1 ratio) gels in $0.5 \times$ Tris-boric acidEDTA (TBE: $90 \mathrm{mM}$ Tris-boric acid and $2 \mathrm{mM}$ EDTA) buffer at $150 \mathrm{~V}$ and $4^{\circ} \mathrm{C}$ for $1.5 \mathrm{~h}$. Gels were then stained with SYBR Green EMSA nucleic acid gel stain (Invitrogen) for $20 \mathrm{~min}$ in $0.5 \times \mathrm{TBE}$, and DNA was visualized under UV light.

$K_{D}$ measurements by microcalorimetry. ITC experiments were performed at $20^{\circ} \mathrm{C}$ with an ITC200 isothermal titration calorimeter from MicroCal (GE Healthcare). Protein concentration, in terms of the monomer in the microcalorimeter cell $(0.2 \mathrm{ml})$, was $50 \mu \mathrm{M}$. Nineteen $2-\mu \mathrm{l}$ injections of DNA fragment solution at $500 \mu \mathrm{M}$ were performed at 180 -s intervals, with stirring at 1,000 rpm. Experimental data were fitted to theoretical titration curves with the software program supplied by MicroCal (Origin). This program uses the relation between the heat generated by each injection and $\Delta \mathrm{H}$ (enthalpy changes in Kcal $\mathrm{mol}^{-1}$ ), $\mathrm{Ka}$ (the association binding constant in $\mathrm{M}^{-1}$ ), $n$ (the number of binding sites), total protein concentration, and free and total ligand concentrations.

HPLC analyses. The chemical reagents (solvent and standards) were purchased from VWR (Leuven, Belgium) and Interchim (Montluçon, France). HPLC analyses were used to monitor HCA degradation, as previously described (Campillo et al. 2014), using an Agilent 1200 series HPLC system (Agilent Technologies, Santa Clara, CA) coupled to a UV-visible Diode Array Detector (Agilent Technologies) equipped with a Kromasil $100-5 \mathrm{C}_{18}$ column $(250 \mathrm{~nm}$ by $4.6 \mathrm{~mm}, 5 \mu \mathrm{m}$; AkzoNobel, Bohus, Sweden). The HCA elution gradient $\left(\mathrm{MeOH} / \mathrm{H}_{2} \mathrm{O}\right)$ was performed according to Campillo et al. (2014). UV-vis spectra and the retention times of commercial standards for all HCA were also analyzed as controls. After $8 \mathrm{~h}$ of incubation, the percentage of degraded HCA was calculated by dividing the quantity of HCA measured in culture supernatant by the quantity present in the initial culture medium $\left(\mathrm{T}_{0}=100 \%\right.$ of the HCA). Standard deviations were obtained from three technical and two biological replicates.

Plant inoculation and quantification of bacterial colonization. For rhizospheric studies, tomato seed (Solanum lycopersicum) were sterilized according to Vacheron et al. (2016). Seed were scarified, inoculated with $10 \mu$ l of overnight culture $\left(10^{6}\right.$ $\mathrm{CFU} / \mathrm{ml}$ ) of the appropriate strains, and grown in a climatic chamber with $18 \mathrm{~h}$ of light and $6 \mathrm{~h}$ of darkness on plates containing $0.8 \%$ agar plant cell culture supplemented with a nutrient solution (Plant-Prod 15-15-30 High K; Master Plant-Prod Inc., Brampton, ON Canada) at $1.5 \mathrm{~g} /$ liter. For in planta pathogenic studies, an incision was made with a scalpel blade in the stems of 3 -week-old tomato plants cultivated with a photoperiod of $18 \mathrm{~h}$ of light and $6 \mathrm{~h}$ of darkness in a greenhouse, and then the plants were inoculated with $10 \mu \mathrm{l}$ of overnight culture $\left(10^{8} \mathrm{CFU} / \mathrm{ml}\right)$ of the appropriate strains. 
To determine bacterial colonization levels, roots and tumors were first ground 2 and 3 weeks after inoculation, respectively. Serial dilutions were plated onto 1A medium (tumor) or YPG medium (roots) using a spiral plater (EasySpiral; Interscience, Saint-Nom-la-Bretèche, France). After $48 \mathrm{~h}$ of incubation at $28^{\circ} \mathrm{C}$, colonies were counted using a Scan 1200 camera coupled with a computer system for CFU determination. Colonization results (six plants per strain, with enumeration of four Petri dishes for each plant) were log transformed and compared with the Mann-Whitney-Wilcoxon test $(P=0.05)$, using the R v3.1.3 statistical software environment (R Core Team 2014).

CI were determined using the hcaR kanamycin-sensitive mutant and a kanamycin-resistant derivative of the C58 (pTiatu6148:Km wild-type strain) (Lang et al. 2013). Strains were mixed in $1: 1$ volumes $\left(10^{8} \mathrm{CFU} / \mathrm{ml}\right.$ each), $10 \mu \mathrm{l}$ was inoculated into wounded tomato stems or seed, and $100 \mu \mathrm{l}$ was used to inoculate YPG medium for in vitro competition assays. The input ratio of the initial inoculum was determined by plating the initial inoculum with a spiral plater (EasySpiral; Interscience) onto YPG agar medium. In vitro competition assays were stopped after 1 day of incubation and in planta competition assays (tomato roots) 2 weeks after inoculation. Serial dilutions of the culture medium (in vitro output) or ground roots (in planta output) were plated on YPG medium. Tumor competition assays (in planta output) were stopped 3 weeks after inoculation; tumors were ground and serial dilutions were plated onto $1 \mathrm{~A}$ medium. For each condition (initial inoculum, in vitro output, and in planta output), 100 colonies were transferred onto YPG medium containing kanamycin and neomycin (each at $25 \mu \mathrm{g} / \mathrm{ml}$ ), in order to determine the relative proportions of the two strains. Three independent assays were performed with eight plants each for in planta competition assays and four technical replicates for in vitro competition assays. CI were calculated as described by Bäumler et al. (1997). Briefly, the C584hcaR/C58 ratio of each in vitro or in planta output sample was divided by the corresponding input ratio in the initial inoculum. Each CI condition was analyzed using the Mann-Whitney-Wilcoxon test $(P=0.05)$ to test whether CI values differed according to each condition (in vitro, tumor, and tomato rhizosphere).

Confocal microscopy analyses. A confocal laser-scanning microscope (LSM 800 Meta Confocal Microscope; Zeiss, Oberkochen, Germany) was used to visualize the expression of the Patu1416 and Pvirb e-gfp-transcriptional fusions harbored by the C58 and C584hcaR strains on tomato roots and tumors. Patu1416 and Pvirb expression were analyzed at 14 and 2 days postinoculation, respectively. Thin tomato root sections and young tumor tissue were mounted between a slide and a coverslip in commercial mounting fluid (Aqua Poly/Mount; Polysciences, Inc., Warrington, PA). eGFP and M-cherry were excited with an argon laser at 488 and $584 \mathrm{~nm}$, respectively, and fluorescence was monitored at $528 \mathrm{~nm}$ and $607 \mathrm{~nm}$. M-cherry (constitutive expression) reported the presence of bacteria and eGFP reported gene expression. Images were recorded using LSM 800 software (Zeiss). For each image, the arithmetic mean intensity of M-cherry and eGFP were obtained using LSM 800 software. The arithmetic means eGFP fluorescence intensity was corrected by that of M-cherry. Comparison of the expression was performed by the Mann-Whitney-Wilcoxon test $(P=$ 0.05). Standard deviation was obtained from three biological replicates and two independent experiments.

\section{ACKNOWLEDGEMENTS}

We thank L. Loiseau (PARMIC platform of UMR 5557, Ecologie Microbienne, INRA, UMR 1418) for technical assistance and A. Buchwalter for reading the manuscript and providing suggestions.

\section{LITERATURE CITED}

Allaway, D., Schofield, N. A., Leonard, M. E., Gilardoni, L., Finan, T. M., and Poole, P. S. 2001. Use of differential fluorescence induction and optical trapping to isolate environmentally induced genes. Environ. Microbiol. 3:397-406.

Andersson, D. I., and Hughes, D. 2010. Antibiotic resistance and its cost: Is it possible to reverse resistance? Nat. Rev. Microbiol. 8:260-271.

Ashby, A. M., Watson, M. D., Loake, G. J., and Shaw, C. H. 1988. Ti plasmid-specified chemotaxis of Agrobacterium tumefaciens C58C1 toward vir-inducing phenolic compounds and soluble factors from monocotyledonous and dicotyledonous plants. J. Bacteriol. 170: 4181-4187.

Baude, J., Vial, L., Villard, C., Campillo, T., Lavire, C., Nesme, X., and Hommais, F. 2016. Coordinated regulation of species-specific hydroxycinnamic acid degradation and siderophore biosynthesis pathways in Agrobacterium fabrum. Appl. Environ. Microbiol. 82:3515-3524.

Bäumler, A. J., Tsolis, R. M., Valentine, P. J., Ficht, T. A., and Heffron, F. 1997. Synergistic effect of mutations in invA and $l p f C$ on the ability of Salmonella typhimurium to cause murine typhoid. Infect. Immun. 65: 2254-2259.

Bhattacharya, A., Sood, P., and Citovsky, V. 2010. The roles of plant phenolics in defence and communication during Agrobacterium and Rhizobium infection. Mol. Plant Pathol. 11:705-719.

Bolton, G. W., Nester, E. W., and Gordon, M. P. 1986. Plant phenolic compounds induce expression of the Agrobacterium tumefaciens loci needed for virulence. Science 232:983-985.

Brencic, A., Eberhard, A., and Winans, S. C. 2004. Signal quenching, detoxification and mineralization of vir gene-inducing phenolics by the VirH2 protein of Agrobacterium tumefaciens. Mol. Microbiol. 51: 1103-1115.

Brooks, A. N., Turkarslan, S., Beer, K. D., Lo, F. Y., and Baliga, N. S. 2011. Adaptation of cells to new environments. Wiley Interdiscip. Rev. Syst. Biol. Med. 3:544-561.

Calisti, C., Ficca, A. G., Barghini, P., and Ruzzi, M. 2008. Regulation of ferulic catabolic genes in Pseudomonas fluorescens BF13: Involvement of a MarR family regulator. Appl. Microbiol. Biotechnol. 80:475-483.

Campillo, T., Lavire, C., Shams, M., Pothier, J. F., and Pulawska, J. 2012. Detection and identification methods and new tests as developed and used in the framework of Cost873 for bacteria pathogenic to stone fruits and nuts tumorigenic Agrobacterium spp. J. Plant Pathol. 94: S1.97-S1.104.

Campillo, T., Renoud, S., Kerzaon, I., Vial, L., Baude, J., Gaillard, V., Bellvert, F., Chamignon, C., Comte, G., Nesme, X., Lavire, C., and Hommais, F. 2014. Analysis of hydroxycinnamic acid degradation in Agrobacterium fabrum reveals a coenzyme A-dependent, beta-oxidative deacetylation pathway. Appl. Environ. Microbiol. 80:3341-3349.

Cases, I., de Lorenzo, V., and Ouzounis, C. A. 2003. Transcription regulation and environmental adaptation in bacteria. Trends Microbiol. 11:248-253.

Deochand, D. K., and Grove, A. 2017. MarR family transcription factors: Dynamic variations on a common scaffold. Crit. Rev. Biochem. Mol. Biol. 52:595-613

Duprey, A., Reverchon, S., and Nasser, W. 2014. Bacterial virulence and Fis: Adapting regulatory networks to the host environment. Trends Microbiol. 22:92-99.

Grove, A. 2017. Regulation of metabolic pathways by MarR family transcription factors. Comput. Struct. Biotechnol. J. 15:366-371.

Guo, M., Huang, Z., and Yang, J. 2017. Is there any crosstalk between the chemotaxis and virulence induction signaling in Agrobacterium tumefaciens? Biotechnol. Adv. 35:505-511.

Habbadi, K., Meyer, T., Vial, L., Gaillard, V., Benkirane, R., Benbouazza, A., Kerzaon, I., Achbani, E. H., and Lavire, C. 2017. Essential oils of Origanum compactum and Thymus vulgaris exert a protective effect against the phytopathogen Allorhizobium vitis. Environ. Sci. Pollut. Res. Int. Published online. https://doi.org/10.1007/s11356-017-1008-9

Heindl, J. E., Wang, Y., Heckel, B. C., Mohari, B., Feirer, N., and Fuqua, C. 2014. Mechanisms and regulation of surface interactions and biofilm formation in Agrobacterium. Front. Plant Sci. 5:176.

Hirakawa, H., Schaefer, A. L., Greenberg, E. P., and Harwood, C. S. 2012. Anaerobic $p$-coumarate degradation by Rhodopseudomonas palustris and identification of CouR, a MarR repressor protein that binds $p$ coumaroyl coenzyme A. J. Bacteriol. 194:1960-1967.

Joubert, P., Beaupère, D., Lelièvre, P., Wadouachi, A., Sangwan, R. S., and Sangwan-Norreel, B. S. 2002. Effects of phenolic compounds on Agrobacterium vir genes and gene transfer induction-A plausible molecular mechanism of phenol binding protein activation. Plant Sci. 162:733-743. 
Kalogeraki, V. S., Zhu, J., Eberhard, A., Madsen, E. L., and Winans, S. C. 1999. The phenolic vir gene inducer ferulic acid is O-demethylated by the VirH2 protein of an Agrobacterium tumefaciens Ti plasmid. Mol. Microbiol. 34:512-522.

Kim, Y., Joachimiak, G., Bigelow, L., Babnigg, G., and Joachimiak, A. 2016. How aromatic compounds block DNA binding of HcaR catabolite regulator. J. Biol. Chem. 291:13243-13256.

Lang, J., Planamente, S., Mondy, S., Dessaux, Y., Moréra, S., and Faure, D. 2013. Concerted transfer of the virulence Ti plasmid and companion At plasmid in the Agrobacterium tumefaciens-induced plant tumour. Mol. Microbiol. 90:1178-1189.

Lassalle, F., Campillo, T., Vial, L., Baude, J., Costechareyre, D., Chapulliot D., Shams, M., Abrouk, D., Lavire, C., Oger-Desfeux, C., Hommais, F., Guéguen, L., Daubin, V., Muller, D., and Nesme, X. 2011. Genomic species are ecological species as revealed by comparative genomics in Agrobacterium tumefaciens. Genome Biol. Evol. 3:762-781.

Lowe, T. M., Ailloud, F., and Allen, C. 2015. Hydroxycinnamic acid degradation, a broadly conserved trait, protects Ralstonia solanacearum from chemical plant defenses and contributes to root colonization and virulence. Mol. Plant-Microbe Interact. 28:286-297.

Mandal, S., Mitra, A., and Mallick, N. 2009. Time course study on accumulation of cell wall-bound phenolics and activities of defense enzymes in tomato roots in relation to Fusarium wilt. World J. Microbiol. Biotechnol. 25:795-802.

McCullen, C. A., and Binns, A. N. 2006. Agrobacterium tumefaciens and plant cell interactions and activities required for interkingdom macromolecular transfer. Annu. Rev. Cell Dev. Biol. 22:101-127.

Merritt, P. M., Danhorn, T., and Fuqua, C. 2007. Motility and chemotaxis in Agrobacterium tumefaciens surface attachment and biofilm formation. J. Bacteriol. 189:8005-8014.

Nester, E. W. 2015. Agrobacterium: Nature's genetic engineer. Front. Plant Sci. 5:730.

Otani, H., Lee, Y.-E., Casabon, I., and Eltis, L. D. 2014. Characterization of p-hydroxycinnamate catabolism in a soil Actinobacterium. J. Bacteriol. 196:4293-4303.

Otani, H., Stogios, P. J., Xu, X., Nocek, B., Li, S.-N., Savchenko, A., and Eltis, L. D. 2016. The activity of CouR, a MarR family transcriptional regulator, is modulated through a novel molecular mechanism. Nucleic Acids Res. 44:595-607.

Pan, C., Hu, Y. L., Jiang, X. N., and Gai, Y. 2015. Cloning, expression, crystallization and crystallographic analysis of CouR from Rhodopseudomonas palustris. Acta Crystallogr. Sect. F Struct. Biol. Commun. 71 1416-1420.

Parke, D. 1995. Supraoperonic clustering of pca genes for catabolism of the phenolic compound protocatechuate in Agrobacterium tumefaciens. J. Bacteriol. 177:3808-3817.
Parke, D. 1997. Acquisition, reorganization, and merger of genes: Novel management of the $\beta$-ketoadipate pathway in Agrobacterium tumefaciens. FEMS Microbiol. Lett. 146:3-12.

Parke, D., and Ornston, L. N. 2003. Hydroxycinnamate (hca) catabolic genes from Acinetobacter sp. strain ADP1 are repressed by HcaR and are induced by hydroxycinnamoyl-coenzyme A thioesters. Appl. Environ. Microbiol. 69:5398-5409.

Perera, I. C., and Grove, A. 2010. Molecular mechanisms of ligandmediated attenuation of DNA binding by MarR family transcriptional regulators. J. Mol. Cell Biol. 2:243-254.

Price, M. N., Deutschbauer, A. M., Skerker, J. M., Wetmore, K. M., Ruths, T., Mar, J. S., Kuehl, J. V., Shao, W., and Arkin, A. P. 2013. Indirect and suboptimal control of gene expression is widespread in bacteria. Mol Syst. Biol. 9:660.

Quandt, J., and Hynes, M. F. 1993. Versatile suicide vectors which allow direct selection for gene replacement in gram-negative bacteria. Gene 127:15-21.

R Core Team. 2014. R: A Language and Environment for Statistical Computing. R Foundation for Statistical Computing, Vienna.

Shams, M., Campillo, T., Lavire, C., Muller, D., Nesme, X., and Vial, L. 2012. Rapid and efficient methods to isolate, type strains and determine species of Agrobacterium spp. in pure culture and complex environments. Pages 3-20 in: Biochemical Testing. InTechOpen, Rijeka, Croatia.

Schick, A., Bailey, S. F., and Kassen, R. 2015. Evolution of fitness tradeoffs in locally adapted populations of Pseudomonas fluorescens. Am. Nat. 186:S48-S59.

Simoh, S., Quintana, N., Kim, H. K., Choi, Y. H., and Verpoorte, R. 2009. Metabolic changes in Agrobacterium tumefaciens-infected Brassica rapa. J. Plant Physiol. 166:1005-1014.

Vacheron, J., Moënne-Loccoz, Y., Dubost, A., Gonçalves-Martins, M. Muller, D., and Prigent-Combaret, C. 2016. Fluorescent pseudomonas strains with only few plant-beneficial properties are favored in the maize rhizosphere. Front. Plant Sci. 7:1212.

Valentini, M., Gonzalez, D., Mavridou, D. A., and Filloux, A. 2018. Lifestyle transitions and adaptive pathogenesis of Pseudomonas aeruginosa. Curr. Opin. Microbiol. 41:15-20.

Vaudequin-Dransart, V., Petit, A., Poncet, C., Ponsonnet, C., Nesme, X., Jones, J. B., Bouzar, H., Chilton, W. S., and Dessaux, Y. 1995. Novel Ti plasmids in Agrobacterium strains isolated from fig tree and chrysanthemum tumors and their opinelike molecules. Mol. Plant-Microbe Interact. 8:311-321.

White, A. P., Sibley, K. A., Sibley, C. D., Wasmuth, J. D., Schaefer, R., Surette, M. G., Edge, T. A., and Neumann, N. F. 2011. Intergenic sequence comparison of Escherichia coli isolates reveals lifestyle adaptations but not host specificity. Appl. Environ. Microbiol. 77: 7620-7632 